KOMPASS

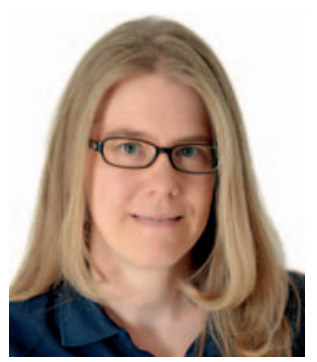

Dr. Anja Gesierich

Klinik und Poliklinik für Dermatologie,

Venerologie und Allergologie

Universitätsklinikum Würzburg

Die Bevölkerung in Deutschland wird immer älter. Laut statistischem Bundesamt werden in 10 Jahren bereits 26\% und in 20 Jahren 30\% der Bevölkerung älter als 65 Jahre sein. Insbesondere dieser Anteil der Bevölkerung hat ein hohes Risiko an Hauttumoren zu erkranken. In einer aktuellen Arbeit von Ulrike Leiter et al. [1] wurde die Inzidenz von epithelialen Hauttumoren (NMSC) anhand von Daten der Krebsregister des Saarlandes und Schleswig- Holsteins untersucht. In den letzten 10 Jahren wurde ein jährlicher Anstieg der rohen Inzidenzrate um 5-7,7\% bei Männern und um 4,4-7,4\% bei Frauen beobachtet. Prognostisch wird bis 2030 für die Altersgruppe $>60$ Jahre mit einem Anstieg der Inzidenzrate um das 2-Fache, insbesondere bei Männern, gerechnet. Dieser Trend wird bei unveränderter UV-Exposition und mangelnder UV-Protektion vermutlich erst ab 2050 stagnieren. So werden wir zukünftig mit einer sehr hohen Anzahl von Hauttumorpatienten in höherem Lebensalter in unseren Arztpraxen, Ambulanzen oder Kliniken konfrontiert werden. Welche Therapie(n) am besten mit den Bedürfnissen und Lebensumständen dieser Patientenklientel in Einklang gebracht werden kann, ist aber bereits jetzt Gegenstand medizinischer, ethischer und gesundheitspolitischer Überlegungen:

\title{
Hauttumoren im Alter - sind wir gewappnet?
}

In dieser Ausgabe des Karger Kompass Dermatologie stellt Dr. Maschke im «Spotlight» zur Onkologischen Dermatochirurgie im Alter fünf hochinteressante Arbeiten vor, die dieses Thema aus verschiedenen Gesichtspunkten beleuchten. Herr Prof. Schilling gibt im Anschluss an dieses Editorial einen Einblick in das Schwerpunktthema dieses Heftes und macht deutlich, welche Aspekte bei der Behandlung von Patienten mit Hauttumoren im Alter beachtet werden sollten.

Neben den invasiven NMSC ist auch mit einer weiter steigenden Zahl von Patienten mit aktinischen Keratosen zu rechnen. Für ihre Behandlung steht uns zwar eine Vielzahl von therapeutischen Möglichkeiten zur Verfügung, vergleichende Studien fehlten aber bisher. Einen Einblick in diese Problematik der geeigneten Therapiewahl sowie einige Eindrücke hierzu liefern zwei Wissenstransfers zu vergleichenden therapeutischen Ansätzen: Prof. Lehmann stellt in seinem Wissenstransfer eine Studie vor, die die klassische PDT mit MethylaminoLävolinsäure (MAL-PDT) versus Ingenolmebutat Gel vergleicht. Dr. Braun-Falco diskutiert zudem die Daten einer Studie, die die läsionale MAL-PDT mit der Flächentherapie vergleicht.
Auch die Inzidenz für das Melanom hat sich in Deutschland seit 1970 mehr als verfünffacht. Die Systemtherapie beim metastasierten Melanom wurde in den letzten Jahren revolutioniert, wie Dr. Heppt und Kollegen in der einleitenden Übersichtsarbeit darstellen. Sie geben zusätzlich einen Ausblick auf neue in der klinischen Prüfung befindliche Kombinationen. Aufgrund der relativ guten Verträglichkeit können auch immer mehr ältere Patienten von diesen neuen Therapieoptionen profitieren. Nicht zuletzt stellen Melanome an ungewöhnlichen Lokalisationen, wie z.B. der Schleimhaut, die Mediziner vor Herausforderungen, wie der Fall von Martinelli-Kläy et al. zeigt. Im Lichte der Aspekte, die in dieser Ausgabe von Karger Kompass Dermatologie vorgestellt und diskutiert werden, und vor dem Hintergrund der älter werdenden Bevölkerung und weiter steigenden Inzidenz von Hauttumoren wird es immer dringlicher, sich mit den besonderen Bedürfnissen dieses Patientenkollektivs auseinanderzusetzen.

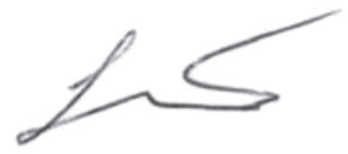

Dr. Anja Gesierich

\section{Literatur}

1 Leiter U, Keim U, Eigentler T, et al.: Incidence, mortality and trends of non-melanoma skin cancer in Germany. J Invest Dermatol 2017; DOI:10.1016/j.jid.2017.04.020.

\section{KARGER}

Fax +497614520714 information@karger.com www.karger.com (c) 2017 S. Karger GmbH, Freiburg
Dr. Anja Gesierich

Klinik und Poliklinik für Dermatologie, Venerologie und Allergologie Universitätsklinikum Würzburg

Josef-Schneider-Straße 2, Gebäude D8, 97080 Würzburg, Deutschland Gesierich_A@ukw.de 


\section{Hauttumoren im Alter}

\section{Epidemiologie}

Epitheliale Hauttumoren stellen die häufigste Krebsform beim Menschen dar. In Deutschland ist eine Steigerung der Inzidenz und Prävalenz von malignen Hauttumoren zu beobachten [1]. Das Auftreten ist altersabhängig, da die kumulative UV-Belastung wesentlich für die Pathogenese ist. Das Maligne Melanom tritt ebenfalls immer häufiger auf, auch bei Patienten höheren Alters [2]. Die stärkste Steigerung der Inzidenz ist aktuell jedoch für das Merkelzellkarzinom zu beobachten [3]. Die Erkrankung verläuft oft aggressiv, und eine leitliniengerechten Versorgung ist multimodal, weshalb diese stetig wachsende Patientengruppe klinisch zunehmend relevant wird.

\section{Herausforderungen und Handlungsbedarf}

Die zunehmende Lebenserwartung führt zu einer Alterung des dermatoonkologischen Patientenkollektivs. Dies führt zu neuen und zunehmenden Herausforderungen in ambulanter und stationärer Versorgung. Durch die vermeintlich kurze Lebenserwartung ist bei den Betroffenen, Angehörigen und Betreuenden ein Neglect zu beobachten. Eine fachärztliche Untersuchung findet häufig nur verzögert statt, sodass Tumoren öfter lokal fortgeschritten sind [4]. Komorbiditäten wie Herzinsuffizienz, Demenz und Diabetes mellitus schränken die Mobilität der Patienten ein und erhöhen Therapierisiken. Insbesondere die zunehmende Prävalenz von Demenz stellt dabei ein großes Problem dar: Anamnese und Befunderhebung sind eingeschränkt, die Patienteninformation und gemeinsame Therapieentscheidung oft unmöglich [5]. Die mit der gesetzlichen Betreuung bedachten Personen stehen vor der Herausforderung, die Betroffenen in ihrem Sinne zu vertreten.

\section{Therapieoptionen}

Die schnittrandkontrollierte Exzision ist der Goldstandard zur Therapie epithelialer Hauttumoren, auch bei Patienten höheren Alters $[1,6]$. Da sie sehr häufig unter Lokalanästhesie (LA) durchgeführt werden kann, bestehen keine zusätzlichen Narkoserisiken, sodass Tumoren bei frühzeitiger Exzision gut kurativ entfernt werden können. Wird dies jedoch verzögert, kann eine Intubationsnarkose nötig sein, die jedoch durch Begleiterkrankungen eingeschränkt werden kann. Die Betroffenen sind dann der Morbidität durch die Hauttumoren zunehmend ausgesetzt. Bei dementen Patienten kann die Operation unter LA Angst und wehrhaftes Verhalten auslösen, welches die Exzision unmöglich macht. Gleiches gilt für Strahlentherapien, bei denen das Bestrahlungsfeld ruhig gehalten werden muss [7]. Therapieempfehlungen sollten daher nicht allein vom kalendarischen Alter abhängig gemacht werden. Es ist nur ein Faktor der neben Patientenwunsch, Komorbiditäten, körperlicher und seelischer Verfassung und Versorgungssituation die Patientenführung beeinflussen sollte. Erfreulicherweise scheint dies Berücksichtigung zu finden [8]. Das interdisziplinäre Management ist einmal mehr von größter Bedeutung, um auch älteren Patienten mit Hautkrebs eine Teilhabe an einer modernen medizinischen Versorgung zu ermöglichen [9].

\section{Literatur}

1 Leiter U, Gutzmer R, Alter M, et al.: Kutanes Plattenepithelkarzinom. Hautarzt 2016;67:857-866.

2 Hoejberg L, Gad D, Gyldenkerne N, et al.: Trends in melanoma in the elderly in Denmark, 1980-2012. Acta Oncol 2016;55(suppl 1):52-58.

3 Schadendorf D, Lebbé C, Zur Hausen A, et al.: Merkel cell carcinoma: epidemiology, prognosis, therapy and unmet medical needs. Eur J Cancer 2017;71:53-69.

4 Ciocan D, Barbe C, Aubin F, et al.: Distinctive features of melanoma and its management in elderly patients: a population-based study in France. JAMA Dermatol 2013;149:1150-1157.

5 Manganoni AM, Pizzatti L, Pavoni L, et al.: Melanoma in the elderly. Ital Dermatol Venereol 2017;152:203-207.

6 Heppt M, von Braunmühl T, Berking C: Was gibt es Neues zum Basalzellkarzinom? Hautarzt 2016;67:876-883.

7 Valeriani M, Nicosia L, Agolli L, et al.: Mono- and bi-weekly hypofractionated radiation therapy for the treatment of epithelial skin cancer in very elderly patients. Anticancer Res 2017;37:825-830.

8 Linos E, Chren MM, Stijacic Cenzer I, Covinsky KE: Skin cancer in U.S. elderly adults: does life expectancy play a role in treatment decisions? J Am Geriatr Soc 2016;64:1610-1615.

9 Russo AE, Ferraù F, Antonelli G, et al.: Malignant melanoma in elderly patients: biological, surgical and medical issues. Expert Rev Anticancer Ther 2015;15:101-108.

Prof. Dr. Bastian Schilling, Oberarzt, Klinik für Dermatologie, Venerologie, Allergologie, Universitätsklinikum Würzburg 